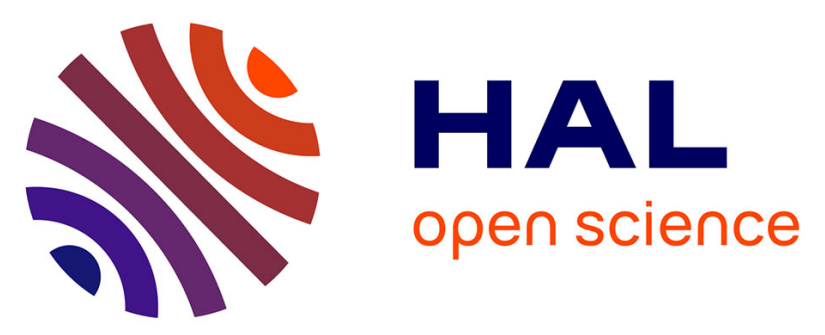

\title{
A Fractional Step Method to Simulate Mixed Flows in Pipes with a Compressible Two-Layer Model
}

Charles Demay, Christian Bourdarias, Benoit de Laage de Meux, Stéphane Gerbi, Jean-Marc Hérard

\section{- To cite this version:}

Charles Demay, Christian Bourdarias, Benoit de Laage de Meux, Stéphane Gerbi, Jean-Marc Hérard. A Fractional Step Method to Simulate Mixed Flows in Pipes with a Compressible Two-Layer Model. Finite Volumes for Complex Applications 8, Jun 2017, Lille, France. pp.33 - 41, 10.1007/978-3-31957394-6_4 . hal-01579379

\section{HAL Id: hal-01579379 \\ https://hal.science/hal-01579379}

Submitted on 31 Aug 2017

HAL is a multi-disciplinary open access archive for the deposit and dissemination of scientific research documents, whether they are published or not. The documents may come from teaching and research institutions in France or abroad, or from public or private research centers.
L'archive ouverte pluridisciplinaire HAL, est destinée au dépôt et à la diffusion de documents scientifiques de niveau recherche, publiés ou non, émanant des établissements d'enseignement et de recherche français ou étrangers, des laboratoires publics ou privés. 


\title{
A fractional step method to simulate mixed flows in pipes with a compressible two-layer model
}

\author{
Charles Demay, Christian Bourdarias, Benoît de Laage de Meux, Stéphane Gerbi \\ and Jean-Marc Hérard
}

\begin{abstract}
The so-called mixed flows in pipes include two-phase stratified regimes as well as single-phase pressurized regimes with transitions. It is proposed to handle those configurations numerically with the compressible two-layer model developed in [7]. Thus, a fractional step method is proposed to deal explicitly with the slow propagation phenomena and implicitly with the fast ones. It results in a large time-step scheme accurate in both regimes. Numerical experiments are performed including convergence results and academical test cases.
\end{abstract}

Key words: Two-layer model, implicit-explicit scheme, mixed flow

\section{Introduction}

We focus on air-water flows in pipes and particularly on the so-called mixed flows. The latter include stratified regimes driven by slow surface waves as well as pressurized regimes (pipe full of water or air) driven by fast acoustic waves. This type of flow occurs in piping systems of several industrial areas such as nuclear and hydraulic power plants or sewage pipelines.

Numerous modelling and numerical issues are tackled when dealing with mixed flows due to the different nature of each regime. Using a 1D approach, a model with an associated numerical scheme is proposed in [1] without computing the air phase. With the aim of accounting for air-water interactions, a compressible two-

Charles Demay ${ }^{1,3}$, Benoît de Laage de Meux ${ }^{1}$, Jean-Marc Hérard ${ }^{1,2}$

(1) EDF Lab Chatou, 6 Quai Watier 78400 Chatou, France;

(2) I2M, UMR 7373-CNRS, Université Aix-Marseille, 13453 Marseille, France;

e-mail: charles.demay@edf.fr, benoit.de-laage-de-meux@edf.fr, jean-marc.herard@edf.fr

Christian Bourdarias ${ }^{3}$, Stéphane Gerbi ${ }^{3}$

(3) LAMA, UMR 5127-CNRS, Université Savoie Mont Blanc, 73376 Le Bourget-du-Lac, France; e-mail: christian.bourdarias@univ-smb.fr, stephane.gerbi@univ-smb.fr 
layer model is developed in [7]. It results in an hyperbolic two-phase two-pressure model which presents strong similarities with the isentropic form of two-fluid models introduced in [3]. In that framework, classical explicit schemes bring large numerical diffusivity in the slow stratified regime.

Thus, a fractional step method is derived herein to split the slow dynamics from the fast dynamics and adapt the numerical treatment. This approach is used in [2,5] for the Baer-Nunziato model and more recently in [6] for the model under consideration. Furthermore, an implicit-explicit time discretization is also proposed in the sequel to end up with a large time-step scheme and get accuracy in the stratified regime. Contrary to the work presented in [6], the overall approach is driven by the fast pressure relaxation and the shallow-water structure of the system such that interesting results are obtained even for low speed flows.

\section{The compressible two-layer model}

The considered model deals with stratified gas-liquid flows in pipes. It results from a depth-averaging of the isentropic Euler set of equations for each phase where the classical hydrostatic assumption is made for the liquid, see [7] for details. Considering a two-layer air-water flow through a pipe of height $H$, it reads:

$$
\left\{\begin{array}{l}
\partial_{t} h_{1}+U_{I} \partial_{x} h_{1}=\lambda_{p}\left(P_{I}-P_{2}\left(\rho_{2}\right)\right), \\
\partial_{t} m_{k}+\partial_{x} m_{k} u_{k}=0 \\
\partial_{t} m_{k} u_{k}+\partial_{x} m_{k} u_{k}^{2}+\partial_{x} h_{k} P_{k}\left(\rho_{k}\right)-P_{I} \partial_{x} h_{k}=(-1)^{k} \lambda_{u}\left(u_{1}-u_{2}\right),
\end{array}\right.
$$

where $k=1$ for water, $k=2$ for air, $m_{k}=h_{k} \rho_{k}$ and $h_{1}+h_{2}=H$. Here, $h_{k}, \rho_{k}, P_{k}\left(\rho_{k}\right)$ and $u_{k}$ denote respectively the height, the mean density, the mean pressure and the mean velocity of phase $k$. The interfacial dynamics is represented by the transport equation on $h_{1}$ while the other two equations account for mass and momentum conservation in each phase. The interfacial pressure is denoted by $P_{I}$ and closed by the hydrostatic constraint, while the interfacial velocity is denoted by $U_{I}$ and closed following an entropy inequality, one obtains (see [7]):

$$
\left(U_{I}, P_{I}\right)=\left(u_{2}, P_{1}\left(\rho_{1}\right)-\rho_{1} g \frac{h_{1}}{2}\right),
$$

where $g$ is the gravity field magnitude. As the phases are compressible, state equations are required for gas and liquid pressures. For instance, perfect gas law may be used for air and linear law for water. The celerity of acoustic waves is defined by $c_{k}=\sqrt{P_{k}^{\prime}\left(\rho_{k}\right)}$. Regarding the source terms, $\lambda_{p}$ and $\lambda_{u}$ are positive bounded functions accounting for relaxation time scales.

\section{Properties of $(\mathscr{S})$}

(i) Smooth solutions of ( $\mathscr{S})$ comply with an entropy inequality. 
(ii) The convective part of (S) is hyperbolic under the condition $\left|u_{1}-u_{2}\right| \neq c_{1}$. Its eigenvalues are unconditionally real and given by $\lambda_{1}=u_{2}, \lambda_{2,3}=u_{1} \pm c_{1}$, $\lambda_{4,5}=u_{2} \pm c_{2}$. The field associated with the 1-wave is linearly degenerate while the other fields are genuinely nonlinear.

(iii) Unique jump conditions hold within each isolated field.

(iv) The positivity of $h_{k}$ and $\rho_{k}$ is verified.

The details and proofs are provided in [7]. Two additional properties of $(\mathscr{S})$ are used in the proposed fractional step method. Firstly, using (1), the momentum equation for water can be written under a Saint-Venant-like form (see [8]):

$$
\partial_{t} m_{1} u_{1}+\partial_{x} m_{1} u_{1}^{2}+\partial_{x} \rho_{1} g \frac{h_{1}^{2}}{2}+h_{1} \partial_{x} P_{I}=\lambda_{u}\left(u_{2}-u_{1}\right) .
$$

Secondly, the pressure relaxation in the first equation of $(\mathscr{S})$ writes classically:

$$
P_{I} \underset{t \rightarrow \infty}{\rightarrow} P_{2}
$$

and this relaxation is very fast in our framework as $\lambda_{p} \gg 1$. In addition, regarding the pressurized regime, $(\mathscr{S})$ degenerates towards a single-phase Euler system when one phase vanishes, as soon as the source terms also vanish.

\section{Fractional step method adapted to mixed flows}

In order to handle both regimes included in mixed flows, the proposed fractional step method splits $(\mathscr{S})$ into three sub-systems. The material component of $(\mathscr{S})$ is treated in $\left(\mathscr{S}_{m}\right)$ including the pressure relaxation source term and using the SaintVenant structure (2) for the water phase:

$$
\left\{\begin{array}{l}
\partial_{t} h_{1}+u_{2} \partial_{x} h_{1}=\lambda_{p}\left(P_{I}-P_{2}\right) \\
\partial_{t} m_{k}+\partial_{x} m_{k} u_{k}=0, k=1,2 \\
\partial_{t} m_{1} u_{1}+\partial_{x} m_{1} u_{1}^{2}+\partial_{x} \rho_{1} g \frac{h_{1}^{2}}{2}=0 \\
\partial_{t} m_{2} u_{2}+\partial_{x} m_{2} u_{2}^{2}=0
\end{array}\right.
$$

$\left(\mathscr{S}_{a}\right)$ refers to the acoustic component of $(\mathscr{S})$ including the pressure gradients:

$$
\left\{\begin{array}{l}
\partial_{t} h_{k}=0, \partial_{t} m_{k}=0, k=1,2, \\
\partial_{t} m_{1} u_{1}+h_{1} \partial_{x} P_{I}=0, \\
\partial_{t} m_{2} u_{2}+h_{2} \partial_{x} P_{2}+\left(P_{2}-P_{I}\right) \partial_{x} h_{2}=0,
\end{array}\right.
$$

where $P_{I}=P_{1}\left(\rho_{1}\right)-\rho_{1} g \frac{h_{1}}{2}$. Finally, $\left(\mathscr{S}_{u}\right)$ deals with the velocity relaxation source terms:

$$
\partial_{t} h_{k}=0, \partial_{t} m_{k}=0, \partial_{t} m_{k} u_{k}=(-1)^{k} \lambda_{u}\left(u_{1}-u_{2}\right), k=1,2 .
$$


A key feature is that the fast relaxation (3) solved in $\left(\mathscr{S}_{m}\right)$ is explicitly seen by $\left(\mathscr{S}_{a}\right)$.

Proposition 1 (Hyperbolicity of $\left(\mathscr{S}_{m}\right)$ ). The convective part of $\left(\mathscr{S}_{m}\right)$ is weakly hyperbolic. Its eigenvalues are given by $\left\{u_{2} ; u_{1} \pm \sqrt{g \frac{h_{1}}{2}}\right\}$.

$\left(\mathscr{S}_{a}\right)$ is not hyperbolic as its spectrum reduces to zero. This singularity is handled in the sequel using a relaxation approach.

In the discrete setting, the time step is denoted $\Delta t$ and the space step $\Delta x$. The space is partitioned into cells $C_{i}=\left[x_{i-\frac{1}{2}}, x_{i+\frac{1}{2}}\right.$ [ where $x_{i+\frac{1}{2}}=\left(i+\frac{1}{2}\right) \Delta x$ are the cell interfaces. At times $t^{n}=n \Delta t$, the solution is approximated on each cell $C_{i}$ by $\mathbf{W}_{i}^{n}=\left(\left(h_{1}\right)_{i}^{n},\left(h_{1} \rho_{1}\right)_{i}^{n},\left(h_{2} \rho_{2}\right)_{i}^{n},\left(h_{1} \rho_{1} u_{1}\right)_{i}^{n},\left(h_{2} \rho_{2} u_{2}\right)_{i}^{n}\right)^{T}$.

Step 1: Explicit scheme for $\left(\mathscr{S}_{m}\right)$. In this step, $W_{i}$ is updated from $W_{i}^{n}$ to $W_{i}^{*}$. A classical explicit finite-volume scheme with Rusanov fluxes is used on the convective part while the pressure relaxation source term is treated implicitly. It writes:

$$
\mathbf{W}_{i}^{*}=\mathbf{W}_{i}^{n}-\frac{\Delta t}{\Delta x}\left(\mathbf{F}\left(\mathbf{W}_{i+\frac{1}{2}}^{n}\right)-\mathbf{F}\left(\mathbf{W}_{i-\frac{1}{2}}^{n}\right)\right)-\frac{\Delta t}{\Delta x} \mathbf{B}\left(\mathbf{W}_{i}^{n}\right)\left(\mathbf{W}_{i+1}^{n}-\mathbf{W}_{i}^{n}\right)+\mathbf{S}\left(\mathbf{W}_{i}^{*}\right),
$$

where $\mathbf{F}(\mathbf{W})=\left(0, m_{1} u_{1}, m_{2} u_{2}, m_{1} u_{1}^{2}+m_{1} g \frac{h_{1}}{2}, m_{2} u_{2}^{2}\right)^{T}, \mathbf{B}(\mathbf{W})=\left(u_{2}, 0,0,0,0\right)^{T}$ and $\mathbf{S}(\mathbf{W})=\left(\lambda_{p}\left(P_{I}-P_{2}\right), 0,0,0,0\right)^{T}$. The fluxes are defined by:

$$
\left\{\begin{array}{l}
\mathbf{F}\left(\mathbf{W}_{i+\frac{1}{2}}^{n}\right)=\frac{1}{2}\left(\left(\mathbf{W}_{i}^{n}+\mathbf{W}_{i+1}^{n}\right)-r_{i+\frac{1}{2}}\left(\mathbf{W}_{i+1}^{n}-\mathbf{W}_{i}^{n}\right)\right), \\
r_{i+\frac{1}{2}}=\max _{j \in\{i ; i+1\}}\left(\left|u_{2, j}^{n}\right| ;\left|\left(u_{1}+\sqrt{g \frac{h_{1}}{2}}\right)_{j}^{n}\right|\right) .
\end{array}\right.
$$

In order to solve implicitly the source term, the mass terms $m_{k, i}^{n}$ are updated first and the first equation in $\left(\mathscr{S}_{m}\right)$ is solved under the form $f\left(h_{1, i}^{*}\right)=0$ where:

$$
f(y)=y-h_{1, i}^{n}+\Delta t \int_{x_{i-\frac{1}{2}}}^{x_{i+\frac{1}{2}}} u_{2}^{n} \frac{\partial h_{1}^{n}}{\partial x} d x-\Delta t \lambda_{p, i}^{n}\left(P_{I}\left(\frac{m_{1, i}^{*}}{y}\right)-P_{2}\left(\frac{m_{2, i}^{*}}{H-y}\right)\right) .
$$

One may easily demonstrate that $f$ is strictly increasing on $[0 ; H]$ with the limits $f \underset{0^{+}}{\rightarrow}-\infty$ and $f \underset{H^{-}}{\rightarrow}+\infty$, such that $f(x)=0$ admits a unique solution $h_{1, i}^{*}$ on $[0 ; H]$.

Proposition 2 (Positivity of heights and densities). The proposed scheme for $\left(\mathscr{S}_{m}\right)$ ensures the positivity of heights and densities under the classical CFL condition:

$$
\frac{\Delta t}{\Delta x} \max _{i}\left(\frac{r_{i+\frac{1}{2}}+r_{i-\frac{1}{2}}}{2}\right)<1,
$$

which only implies material velocities.

Step 2: Implicit relaxation approach for $\left(\mathscr{S}_{a}\right)$. In this step, only $u_{k}$ is updated from $u_{k}^{*}$ to $u_{k}^{* *}$. The lack of hyperbolicity is handled with a relaxation approach, see 
[4, 5], introducing the system $\left(\mathscr{S}_{a}^{r}\right)$ which relaxes towards $\left(\mathscr{S}_{a}\right)$ in the limit $\varepsilon \rightarrow 0$ :

$$
\left\{\begin{array}{l}
\partial_{t} h_{k}=0, \partial_{t} m_{k}=0, k=1,2, \\
\partial_{t} m_{1} u_{1}+h_{1} \partial_{x} \Pi_{I}=0, \\
\partial_{t} m_{2} u_{2}+h_{2} \partial_{x} \Pi_{2}+\left(\Pi_{2}-\Pi_{I}\right) \partial_{x} h_{2}=0, \\
\partial_{t} m_{k} \Pi_{k}+a_{k}^{2} h_{k} \partial_{x} u_{k}+a_{k}^{2}\left(u_{k}-u_{2}\right) \partial_{x} h_{k}=\frac{1}{\varepsilon} m_{k}\left(\Pi_{k}-P_{k}\right), k=1,2,
\end{array}\right.
$$

where $\Pi_{I}=\Pi_{1}-\rho_{1} g \frac{h_{1}}{2}$ and $\Pi_{k}$ relaxing toward $P_{k}$ as $\varepsilon \rightarrow 0$. The PDE verified by $\Pi_{k}$ is derived from the PDE verified by $P_{k}$ in $(\mathscr{S})$. In addition, $a_{k}$ are positive numerical parameters used to ensure the stability of the relaxation approximation in the regime of small $\varepsilon$, their definition is provided later according to the flow regime.

Proposition 3 (Hyperbolicity of $\left(\mathscr{S}_{a}^{r}\right)$ ). When $a_{k}>0$, the convective part of $\left(\mathscr{S}_{a}^{r}\right)$ is strictly hyperbolic. Its eigenvalues are given by $\left\{0 ; \pm \frac{a_{1}}{\rho_{1}} ; \pm \frac{a_{2}}{\rho_{2}}\right\}$.

In order to keep a numerical diffusivity based on the material CFL condition (7), an implicit-explicit time discretization is proposed for the convective part of $\left(\mathscr{S}_{a}^{r}\right)$ :

$$
\left\{\begin{array}{l}
h_{k}^{* *}=h_{k}^{*}, m_{k}^{* *}=m_{k}^{*}, k=1,2, \\
\left(m_{1}^{* *} u_{1}^{* *}-m_{1}^{*} u_{1}^{*}\right) / \Delta t+h_{1}^{* *} \partial_{x} \Pi_{I}^{* *}=0, \\
\left(m_{2}^{* *} u_{2}^{* *}-m_{2}^{*} u_{2}^{*}\right) / \Delta t+h_{2}^{* *} \partial_{x} \Pi_{2}^{* *}+\left(\Pi_{2}^{*}-\Pi_{I}^{*}\right) \partial_{x} h_{2}^{*}=0, \\
\left(m_{k}^{* *} \Pi_{k}^{* *}-m_{k}^{*} \Pi_{k}^{*}\right) / \Delta t+a_{k}^{2^{* *}} h_{k}^{* *} \partial_{x} u_{k}^{* *}+a_{k}^{2^{*}}\left(u_{k}^{*}-u_{2}^{*}\right) \partial_{x} h_{k}^{*}=0, k=1,2 .
\end{array}\right.
$$

Classical combinations on (8) lead to the following semi-discrete equations on $u_{k}$ :

$$
\left\{\begin{array}{l}
\frac{u_{1}^{* *}-u_{1}^{*}}{\Delta t}-\frac{\Delta t}{\rho_{1}^{*}} \partial_{x}\left(\frac{a_{1}^{2^{*}}}{\rho_{1}^{*}} \partial_{x} u_{1}^{* *}\right)=-\frac{1}{\rho_{1}^{*}} \partial_{x} P_{I}^{*}+\frac{\Delta t}{\rho_{1}^{*}} \partial_{x}\left(\frac{a_{1}^{2^{*}}\left(u_{1}^{*}-u_{2}^{*}\right)}{m_{1}^{*}} \partial_{x} h_{1}^{*}\right), \\
\frac{u_{2}^{* *}-u_{2}^{*}}{\Delta t}-\frac{\Delta t}{\rho_{2}^{*}} \partial_{x}\left(\frac{a_{2}^{2}}{\rho_{2}^{*}} \partial_{x} u_{2}^{* *}\right)=-\frac{1}{\rho_{2}^{*}} \partial_{x} P_{2}^{*}-\frac{\left(P_{2}^{*}-P_{I}^{*}\right)}{m_{2}^{*}} \partial_{x} h_{2}^{*} .
\end{array}\right.
$$

In (9), instantaneous relaxation $(\varepsilon \rightarrow 0)$ is assumed between $\Pi_{k}$ and $P_{k}$ such that $\Pi_{k}^{*}=P_{k}^{*}$. Thus, the proposed implicit relaxation approach acts as a stabilization process involving a diffusion term weighted by $a_{k}$.

Definition 1. Under the light of (9), $a_{k}$ is defined according to the flow regime:

- In the stratified regime $\left(h_{1}<H\right)$ : the pressure gradient $h_{1} \partial_{x} P_{I}$ in $\left(\mathscr{S}_{a}\right)$ is seen as a source term. It accounts for variable interfacial pressure which can be interpreted as air phase pressure due to the relaxation (3) solved in the first step. Thus, $a_{1}$ is set to zero.

- In the pressurized regime $\left(h_{1}=H\right)$ : the stabilization process is applied and $a_{1}$ must follow the so-called Whitham condition: $a_{1}^{2}>\max _{\rho_{1}}\left(\rho_{1}^{2} c_{1}^{2}\right)$, see [4, 5].

- In all the regimes, $a_{2}$ follows the Whitham condition $a_{2}^{2}>\max _{\rho_{2}}\left(\rho_{2}^{2} c_{2}^{2}\right)$. 
After integrating (9) on a cell $C_{i}$ and using centered schemes for gradients, one obtains an implicit system which may be written in matrix form:

$$
A_{k}^{*} \mathbb{U}_{k}^{* *}=\mathbb{S}_{k}^{*}
$$

where $A_{k}^{*}$ is a non-singular tridiagonal matrix (M-matrix structure) and $\mathbb{S}_{k}^{*}$ corresponds to the integrated source term. Calculations are not detailed here. In practice, the diffusion coefficient $\left(a_{k}^{2} / \rho_{k}\right)_{i+\frac{1}{2}}^{*}$ is computed using an harmonic average and a threshold on $h_{1}$ is introduced to identify the flow regime.

Step 3: Implicit scheme for $\left(\mathscr{S}_{u}\right)$. In this step, only $u_{k}$ is updated from $u_{k}^{* *}$ to $u_{k}^{n+1}$. The velocity relaxation source term is treated implicitly (except the $\lambda_{u}$ coefficient) such that the following non-singular $2 \times 2$ system is obtained:

$$
\left(\begin{array}{cc}
m_{1, i}^{* *}+\Delta t \lambda_{u, i}^{* *} & -\Delta t \lambda_{u, i}^{* *} \\
-\Delta t \lambda_{u, i}^{* *} & m_{2, i}^{* *}+\Delta t \lambda_{u, i}^{* *}
\end{array}\right)\left(\begin{array}{c}
u_{1, i}^{n+1} \\
u_{2, i}^{n+1}
\end{array}\right)=\left(\begin{array}{c}
\left(m_{1} u_{1}\right)_{i}^{* *} \\
\left(m_{2} u_{2}\right)_{i}^{* *}
\end{array}\right)
$$

This step concludes the overall scheme which ensures the positivity of heights and densities under the material CFL condition (7).

\section{Numerical results}

In this section, the proposed scheme is denoted $\mathrm{SP}_{\mathrm{r}}$ and compared with a classical Rusanov scheme applied on $(\mathscr{S})$ under an acoustic CFL condition.

Riemann problem for the convective part. One considers an analytical solution which contains two shocks for each phase traveling with the fast acoustic waves and a contact discontinuity (slow wave) where $h_{1}$ jumps. Without the pressure relaxation (3), note that $a_{1}$ follows the Whitham condition. Fields are displayed on figure 1 at $T=23 \cdot 10^{-5} s$ with 500 cells. A mesh refinement is also performed to check the numerical convergence of the method.

As expected, the $\mathrm{SP}_{\mathrm{r}}$ scheme is accurate on the slow wave. Regarding the fast waves, it is more diffusive than Rusanov on phase 1 (the fastest) while better results are obtained on phase 2. Indeed, the optimal regime for the Rusanov scheme is on phase 1 with acoustic time steps. Stability and convergence towards relevant shock solutions are obtained with the expected convergence rate $\frac{1}{2}$ due to the contact discontinuity.

Dambreak. The source terms are activated and one considers the dambreak problem where the initial condition is a discontinuity on $h_{1}$ with constant density and zero speed. Regarding the water layer, the (incompressible) Saint-Venant system admits an analytical solution, see [8]. As the compressibility of water as well as the additional air layer should have a minor influence here, one expects to obtain the 

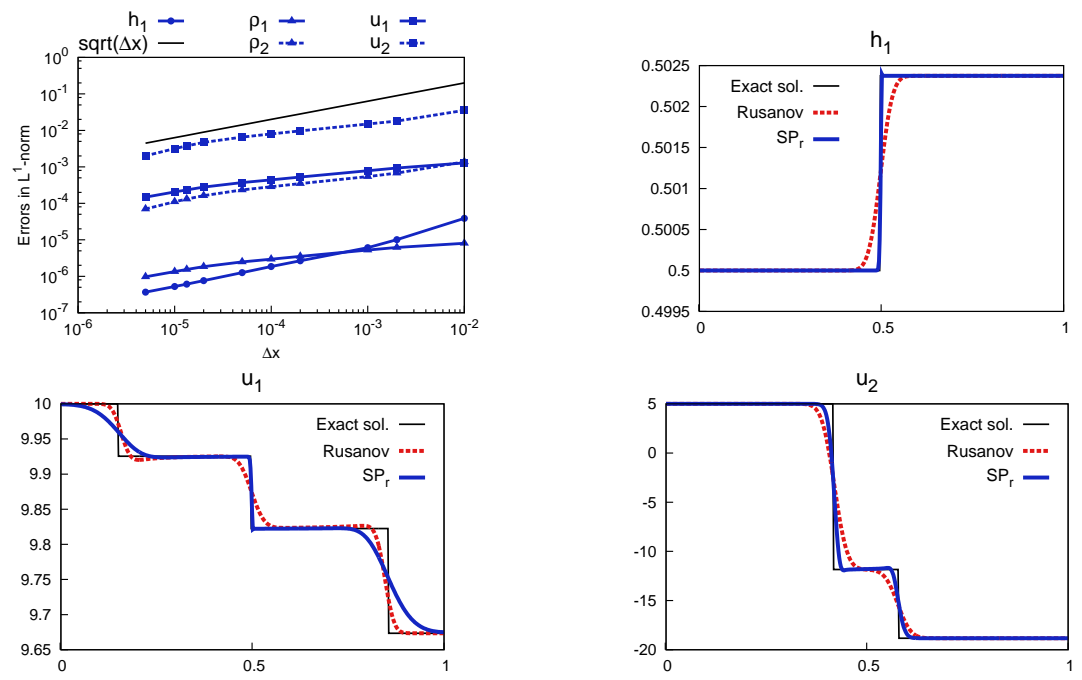

Fig. 1 Errors in $\mathrm{L}^{1}$-norm and fields at $T=23 \cdot 10^{-5} s$ with 500 cells for the Riemann problem
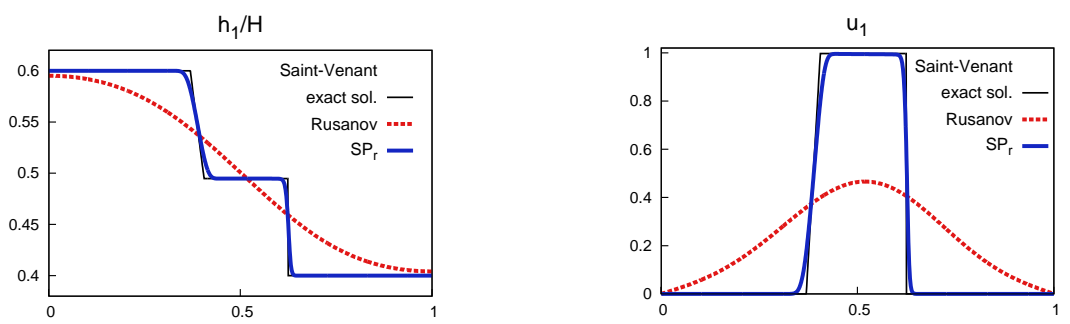

Fig. 2 Fields at $T=24 \cdot 10^{-3} s$ with 1000 cells for the dambreak problem

same kind of solution for phase 1 . Therefore, a $1 \mathrm{~m}$ long pipe is considered with $\left(h_{1} / H\right)_{L}=0.6$ and $\left(h_{1} / H\right)_{R}=0.4$ as initial conditions. The velocity and height fields for phase 1 are plotted on figure 2 at $T=24 \cdot 10^{-3} s$ using 1000 cells.

Contrary to the results obtained with the large time-step scheme proposed in [6], the $\mathrm{SP}_{\mathrm{r}}$ scheme displays accurate fields regarding the Saint-Venant solution. The Rusanov scheme is highly diffusive and regarding CPU time, it needs 3 minutes while $\mathrm{SP}_{\mathrm{r}}$ takes 6 seconds. Those results emphasize the fact that a classical explicit scheme applied on $(\mathscr{S})$ is not adapted to low speed configurations.

Mixed flow. One considers a closed sloping pipe with constant height and zero speed as initial conditions. The pipe is $5 \mathrm{~m}$ long with $H=1 \mathrm{~m}, h_{1}=0.8 \mathrm{~m}, \theta=30$ degrees and a 250 cells mesh is used. The flow becomes pressurized at the bottom (only water) and dried at the top (only air), see figure 3 for a snapshot of the water height and figure 4 for the pressure field. 

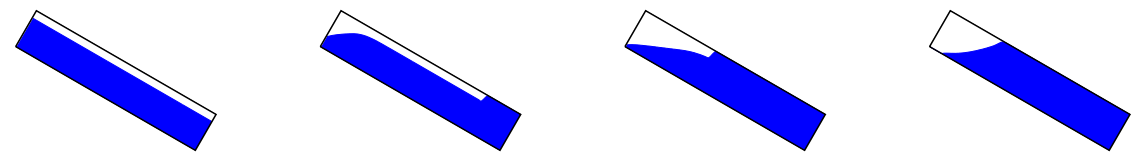

Fig. 3 Pipe filling snapshots for water height with 250 cells

Interesting qualitative results are obtained which demonstrates the ability of the $\mathrm{SP}_{\mathrm{r}}$ scheme to handle mixed flows. Regarding the pressure field, one observes oscillations at the transition point between the regimes which are classical when dealing with mixed flows, see [1]. Further validation is obviously needed using available experimental data.
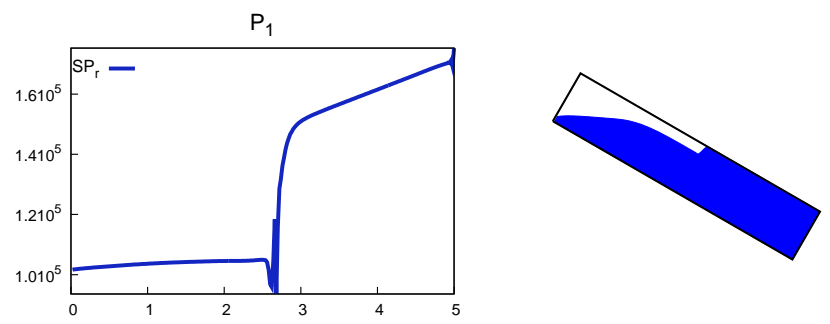

Fig. 4 Pressure field and water height at $T=0.35 \mathrm{~s}$ with 250 cells

Acknowledgements C. Demay received a financial support by ANRT through an EDF-CIFRE contract 2014/749. Numerical facilities were provided by EDF.

\section{References}

1. Bourdarias, C., Gerbi, S.: A finite volume scheme for a model coupling free surface and pressurised flows in pipes. J. Comput. Appl. Math. 209, 1-47 (2007)

2. Chalons, C., Coquel, F., Kokh, S., Spillane, N.: Large time-step numerical scheme for the sevenequation model of compressible two-phase flows. Springer Proceedings in Mathematics and Statistics 4, 225-233 (2011)

3. Coquel, F., Gallouët, T., Hérard, J.M., Seguin, N.: Closure laws for a two-fluid two-pressure model. C. R. Acad. Sci. Paris 334(I), 927-932 (2002)

4. Coquel, F., Godlewski, E., Seguin, N.: Relaxation of fluid systems. Math. Models Methods Appl. Sci. 22(8) (2012)

5. Coquel, F., Hérard, J.M., Saleh, K.: A splitting method for the isentropic Baer-Nunziato twophase flow model. ESAIM: Proceedings 38(3), 241-256 (2012)

6. Demay, C., Bourdarias, C., De Laage de Meux, B., Gerbi, S., Hérard, J.M.: Numerical simulation of a compressible two-layer model: a first attempt with an implicit-explicit splitting scheme. Submitted (2016). URL https://hal.archives-ouvertes.fr/hal-01421889

7. Demay, C., Hérard, J.M.: A compressible two-layer model for transient gas-liquid flows in pipes. Continuum Mech. Thermodyn. (2016). DOI 10.1007/s00161-016-0531-0

8. Gerbeau, J.F., Perthame, B.: Derivation of viscous Saint-Venant system for laminar shallow water; numerical validation. Discrete Contin. Dyn. Syst. Ser. B 1, 89-102 (2001) 